\title{
Teorema de Emmy Nöther, 100 anos: Alegoria da Misoginia em Ciência
}

\author{
Emmy Nöther's Theorem, 100 years: Allegory of the Misogyny in Science
}

\author{
Roberta Areas ${ }^{1,2}$, Marcia C. Barbosa ${ }^{1}$, Ademir E. Santana*3 \\ ${ }^{1}$ Universidade Federal do Rio Grande do Sul, Instituto de Física, Porto Alegre, RS, Brasil \\ ${ }^{2}$ Ministério da Educação, Coordenação de Aperfeiçoamento de Pessoal de Nível Superior, Brasília, DF, Brasil \\ ${ }^{3}$ Universidade de Brasília, Instituto de Física, Centro Internacional de Física, Brasília, DF, Brasil
}

Recebido em 25 de Janeiro, 2019. Revisado em 31 de Março, 2019. Aceito em 02 de Abril, 2019.

\begin{abstract}
O teorema de Nöther é revisitado com dois objetivos: ressaltar a significado desses resultados para a física contemporânea e ao mesmo tempo apresentar aspectos da biografia de Emmy Nöther, enfatizando a misoginia sofrida na sua atividade científica, a despeito da reconhecida importância, ainda em vida, de seus trabalhos. Sob esta perspectiva, a vida de Emmy Nöther levanta a questão sobre a situação atual da atuação da mulher em ciência. Esse aspecto é abordado através de uma análise da participação de mulheres atuando em ciências com dados do CNPq e da CAPES. Para discutir e apresentar a dimensão apropriada dos resultados principais de Emmy Nöther, contextualizando a importância na física contemporânea, um caminho algébrico é seguido (nos livros textos, os teoremas são apresentados através do formalismo Lagrangiano), mas tanto quanto possível procurando uma abordagem discurso pedagógica.
\end{abstract}

Palavras-chave: Teorema de Nöther; Simetrias; Misoginia em ciência; Gênero.

The Nöther's theorem is revisited with two main goals: to reinforce the meaning of such results for the contemporary physics and to present aspects of the Nöther's biography emphasizing the misogyny process that she suffered during her scientific life, despite of the recognized importance of her results even when she was alive. Under this perspective, the Nöther's live raises questions about the present-day participation of women in science. This aspect is addressed by an analysis of female researchers, by using data from CNPq and CAPES (two Brazilian Government agencies). In order to discuss and to present a proper dimension of the Nöther results, emphasizing such results for physics, an algebraic way is followed (usually such results are derived through the Lagrangian formalism); however, as much as possible, looking for a pedagogical presentation.

Keywords: Nöther's theorem; Symmetry; Misogyny in Science; Gender.

\section{Introdução}

Um século se completa desde a proposição e demonstração dos famosos teoremas de Nöther [1]. Não há exagero em se afirmar que o legado da Professora Emmy Nöther sobre simetrias, parcialmente resumido nos famosos teoremas, fundamenta todas as teorias da física moderna, desde o modelo padrão cosmológico, edificado a partir da gravitação Einsteniana, até o modelo padrão da física das partículas elementares, estabelecido através da teoria quântica de campos 2 .

Sobre sua vida e sobre os famosos teoremas muito se tem, apropriadamente, escrito 2 8]; mas um aspecto de sua biografia ainda demanda análise, principalmente quando considerado em perspectiva de cem anos depois: a discriminação de natureza misógina que sofreu Emmy Nöther no decorrer de sua carreira científica. Isto levanta a questão, atualíssima, sobre a participação da mulher nas ciências 9 17. Esses elementos definem a preocupação central deste trabalho de natureza biográfica, mas que, ademais, se presta a homenagear aquela que também é considerada a mãe da álgebra moderna [5].

Em 1919, já amplamente reconhecida nos círculos matemáticos pela genialidade dos seus trabalhos em álgebra, Emmy Nöther candidatou-se e foi aprovada para uma posição acadêmica na Universidade de Göttingen; centro que contava com nomes como David Hilbert, Felix Klein, Albert Einstein, entre outros. Entretanto, mesmo recebendo o apoio desses eminentes nomes, o conservadorismo daquela instituição impediu-lhe a contratação pelo simples e exclusivo pretexto de que se tratava de uma mulher [2].

Há registro de que Hilbert, irritado com aquela decisão, manifestou-se dizendo, em resumo, que Göttingen era uma Universidade, e não uma sala de banhos, onde a questão biológica do sexo poderia vir a ser acompanhada de alguma discussão 2 . Emmy acabou sendo contratada em 1922, mas como professora extraordinária, inicial-

*Endereço de correspondência: asantana@unb.br 
mente sem receber salário. Esse cargo Emmy manteve até 1933, quando teve que deixar a Alemanha nazista.

A história da vida de Emmy Nöther, inclusive por suas posições politizadas, se mistura à luta pela participação efetiva, não discriminada, equânime, da mulher nas sociedades contemporâneas. O processo de construção dessa disparidade, ainda atual, tem sido analisado sob diversas formas. Os fundamentos se encontram na construção social de conceitos como "homem" antagônico à "mulher", que são naturalizados. Entretanto, esta naturalização nada, ou muito pouco, tem na relação com genitálias biológicas, como de certa forma retrata a observação (acima) de Hilbert sobre a não contratação de Emmy.

O problema de gênero atravessou os séculos, persiste, se mostra longe de ser resolvido, e usualmente é abordado a partir de dois pontos de vista interrelacionados: a violência implícita, também chamada de violência simbólica, e a brutalidade explícita contra a mulher.

Em nada perdendo em brutalidade para a violência explícita, a violência implícita, silenciosa e invisível por vezes, é imposta ao ser individual que é acometida desde transtornos psicológicos a frustrações permanentes, dentre outras sequelas 18,19.

A violência muda é marcada pelo princípio da negação das diferenças [20], que conduz a uma condição de naturalização de procedimentos insidiosos. Isso estabelece um desbalanceamento de acúmulo de capital simbólico, que se presta à manutenção das estruturas dos modos de produção sociais fundados, basicamente, na prática do que se denomina de imperialismo cultural e econômico, na perspectiva tanto de classe quanto geopolítica [18,21]. A dinâmica de perpetuação dessa condição reside primariamente em superestruturas como a família, e os temas de natureza explícita vem sendo estudados e discutidos sob muitas perspectivas 22 25]. Os temas de opressão contra as mulheres de natureza implícita [16 26 27], nos quais o imperialismo cultural está atrelado, geralmente se manifesta através de uma falsa naturalização biológica de características da mulher [27]. Em parte, devido às dificuldades teóricas, os desenvolvimentos nesta direção estão a ocorrer, mas em escala muito menor.

A opressão simbólica contra a mulher é traduzida na observação sobre a participação de mulheres em áreas estruturantes do poder nas sociedades modernas 28 30], como as ciências $12,14,31$, sobretudo em física. Vale citar, por exemplo, a porcentagem de profissionais (professores) atuando nas áreas da física em algumas regiões [32]: América do Norte 10\%; América Latina, 12\%; Europa Anglo-Saxônica, 7\%; Europa Latina e Leste Europeu, $22 \%$. Outra área onde esta estruturação do poder se expressa de maneira contundente é a computação. Quando criados, os cursos de computação tinham entre os seus estudantes um número expressivo de mulheres. Nos primórdios, a área necessitava de mão de obra para digitação e assemelhava-se ao serviço braçal de secretária. À medida que a informática tornou-se um instrumento de poder econômico, as mulheres se tornaram minoria. Nos últimos 15 anos o percentual de mulheres em computação decaiu em $10 \%$ [33. A este tipo de constatação, as análises de senso comum revelam apenas uma androcentria que se estabelece no desconhecimento e no preconceito, embota até mesmo os fatos mais transparentes [26, 27], e se mantém pelo menos desde a época da Emmy Nöther.

Devido a atualidade do tipo de brutalidade muda a que foi submetida, a vida de Emmy é exemplo importante para prover uma dimensão mais precisa dessas práticas insidiosas. Partindo da literatura sobre a vida e a importância dos trabalhos de Emmy para matemática e a física, aqui enfatizamos elementos de sua biografia que evidenciam, como exemplo ainda atual, práticas misóginas dentro da academia.

Observa-se que o mesmo padrão de misoginia persistiu, desde Emmy, através do Século XX, quando as biografias de outras fisicistas são consideradas (ver por exemplo a Ref. [9]). O padrão se estabelece pelos procedimentos de naturalização e acúmulo de bens simbólicos dentro da academia, definida por uma estrutura androcêntrica. A perpetuação do padrão pode ser estimada mais acuradamente se uma análise mais atual, mas de carácter coletivo, transcendendo, portanto, a da biografia individual, for implementada. Nesta perspectiva, tendo como referência histórica-alegórica a situação de Emmy, enquanto fisicista do início do século XX, analisamos o conjunto das lideranças científicas no Brasil neste início de século XXI, considerando a distribuição de gênero.

É importante ressaltar ainda que em Emmy, por sua obra e por ser uma sobrevivente dessas práticas insidiosas, encontramos um exemplo do potencial de contribuição efetiva que mulheres ao permanecerem na ciência são capazes de desenvolver. Nesse sentido, a contextualização do teorema de Nöther, em seu significado e na sua relação com todas as áreas da física, incluindo as aplicadas e as de fronteira, deve ser demarcada tanto quanto possível pedagogicamente. Este resultado, dado sua impotância e abrangência, desarticula a argumentação ingênua da naturalização preconceituosa da suposta "incapacidade feminina para áreas como as ciências". Por outro lado, o preconceito sofrido por Emmy não pode ser considerado como caso isolado, pois a existência desse tipo de misoginia apresenta reflexos mensuráveis pela baixa representatividade das mulheres na ciência.

Para conduzir esta análise de conjunto, consideramos inicialmente a distribuição de Bolsas de Pesquisas do CNPq (Conselho Nacional de Pesquisa e Desenvolvimento Tecnológico, agência do Governo Federal Brasileiro), no decorrer de 15 anos. Para ressaltar a importância dos trabalhos de Nöther para a atualidade da física, seus resultados são então revisitados a partir de uma perspectiva algébrica. Essa importância pode ser depreendida como uma medida da misoginia a que foi submetida.

O trabalho está apresentado da seguinte maneira. Na seção 2, uma breve biografia de Emmy Nöther é apresentada, destacando pontos que serão comparados, na seção 3 , com a situação atual de participação de mulheres nas 
ciências. Na seção 4, os resultados de Nöther são discutidos no âmbito da física contemporânea. As conclusões finais estão apresentadas na seção 5

\section{Emmy Nöther e a Academia Androcêntrica}

Amalie Emily Nöther, carinhosamente chamada por Emmy, nasceu em 23 de março de 1882, em Erlangen, cidade universitária desde o ano de 1002, na Alemanha. Era filha de Ida Amalia Kauffmann e Max Nöther; este, um algebrista e professor da Universidade de Erlangen, era ativo pesquisador de uma forte escola de matemática liderada por Felix Klein. Emmy teve dois irmãos que se tornaram também pesquisadores de sucesso. Inicialmente, Emmy, como era prática das moças de famílias burguesas na época, dedicou-se ao piano e ao estudo de Inglês e Francês, sendo diplomada como professora nessas línguas. Em 1900 as universidades alemãs abriram suas portas para a formação de mulheres, mas de modo condicional. No caso de Emmy, ela poderia assistir as aulas, mas somente se os professores concordassem. Quando ingressou, eram somente duas entre 986 estudantes. Apesar de não ser permitido que se matriculasse (poderia somente assistir às aulas), Emmy consegue ser aprovada no exame de graduação [34.

Em 1903 é aprovada para a Universidade de Göttingen. A partir de então passa a dedicar-se a matemática, usufruindo da formação e do contato familiar, enquanto um ambiente intelectualmente propício ao debate matemático; e se dedica em particular à geometria e a álgebra. Em 1904 inscreveu-se como estudante de doutorado na Universidade de Erlangen. Defendeu a tese, aprovada com distinção, sobre teoria dos invariantes algébricos. Emmy foi orientada por Paul A. Gordon e a tese foi intitulada "Sobre Sistemas Completos de Invariantes para Formas Biquadradas Ternárias" [1]. Depois do doutorado, permaneceu em Erlangen, fazendo pesquisa, orientando estudantes e ocasionalmente substituindo docentes sem salário.

Emmy ganha notoriedade com seus resultados, e em 1915 é convidada por Klein e Hilbert para a Universidade de Göttingen, onde estavam a trabalhar com vários aspectos matemáticos da teoria da relatividade geral de Einstein. Para que pudesse assumir independentemente as aulas deveria passar por um processo de Habilitation. Muitos professores daquela universidade se opunham a mulheres ministrarem aulas; e assim, mesmo tendo sido aprovada por sua qualificação matemática, não foi permitido que Emmy realizasse o exame, o que ocorre somente em 1919. Aulas eram-lhe permitido ministrar na universidade, mas ainda sem salário. Hilbert escreve ao governo alemão dizendo temer que Emmy, por não ter salário, fosse para outra universidade. O governo responde que ela não poderia ir para nenhuma outra universidade na Alemanha, pois em todas era proibido para as mulheres darem aula [8]. Em 1922, a despeito do reconhecimento, foi admitida como professora não oficializada, e mais tarde com a incumbência de ensino em álgebra, o que lhe permitia receber um pequeno salário. Assim de 1922 a 1933 Emmy foi Professora da Universidade de Göttinger. Ainda nessa condição, de 1928 a 1929, assume a posição da Professora visitante da Universidade de Moscou [2].

O ambiente na Universidade de Göttinger naqueles anos era de extrema atividade intelectual em física e em física-matemática. Sob a liderança de Emmy, que passou a trabalhar tanto individualmente, quanto com colaboradores e orientandos, o panorama do estudo da álgebra ganhou novos contornos. Naqueles anos a álgebra expande-se para estruturas abstratas como ideias, corpos, e anéis, que se tornam construtos básicos de muitos outros tópicos matemáticos 5]. O trabalho de Emmy passa, então, a influenciar em significativa importância no desenvolvimento da teoria da relatividade geral de Einstein, que colaborava com Klein e Hilbert. É neste período que Emmy demostra o que veio a ser chamado de os "Teoremas de Nöther"; teoremas enunciados em 1915 e publicados em 1918. O trabalho fora apresentado por Klein, ver Figura 1 [2,7].

Em 1933, com a ascensão do nazismo na Alemanha, Emmy, por ser filha de mãe judia e pela proximidade com os físicos soviéticos, é expulsa da universidade e, segue para o exílio, nos Estados Unidos. Inicialmente é contratada para trabalhar como professora do Bryn Mawr College, na Pensilvânia. Após algum tempo, e devido ao seu reconhecido mérito, torna-se pesquisadora do Instituto de Estudos Avançados de Princeton, para onde havia seguido alguns de seus colegas do período de Göttingen, como Einstein, Weyl e Klein.

A ida de Emmy para os EUA não foi também tão simples, e alguns creditam isso ao fato de ser mulher. Por exemplo, Emmy era tão ou mais reconhecidamente famosa, que seus colegas homens que deixaram a Alemanha e foram diretamente para Princeton. A Diretora do Bryn Mawr College, uma ex-colega de Emmy em Göttinger e outros, como Einstein e Weyl, são quem intercederam para a transferência de Emmy para Princeton.

Ovacionada como uma das mais brilhantes mentes matemáticas de todos os tempos, Emmy falece prematuramente em 14 de abril de 1935 .

\section{Invariante Variationsprobleme.}

\section{(F. Klein zum fünfzigjährigen Doktorjubiläum.) \\ Von \\ Emmy Noether in Göttingen.}

Vorgelegt-von F. Klein in der Sitzung vom 26. Juli 1918 ${ }^{1}$ ).

Es handelt sich um Variationsprobleme, die eine kontinuierliche Gruppe (im Lieschen Sinne) gestatten; die daraus sich ergebenden Folgerungen für die zugehörigen Differentialgleichungen finden ihren allgemeinsten Ausdruck in den in $\S 1$ formulierten, in den folgenden Paragraphen bewiesenen Sätzen. Über diese ans

Figura 1: Trabalho de Emmy Nöther, apresentado por Klein. 


\section{A Androcentria na Academia Atual}

Não obstante o enorme reconhecimento da importância do trabalho de Emmy para a física e matemática, muitos dos físicos e matemáticos, talvez por utilizarem sempre o sobrenome "Nöther", ao se referir aos famosos teoremas em física e aos trabalhos em álgebras, consideram esse "Nöther" como sendo um homem. Em outro dito, nem mesmo por todo esse reconhecimento, a ação misógena, quase invisível, deixou de existir. Em certo sentido, quanto mais reconhecidos se tornaram os resultados de Emmy, menos reconhecida passou a ser sua natureza gentil e brilhante e sua feminilidade. No âmbito da comunidade da física e da matemática, fica sub judice, inclusive do ponto de vista moral, a postura de muitos físicos e matemáticos. Vale ressaltar que mesmo em alguns casos em que a legislação não favorece a androcentria, as ações misóginas aumentaram, tanto em valores absolutos quanto relativos 32 . E isso aponta para um processo de estudo e análise permanente quanto a questão de gênero em ciência.

A situação de Emmy Nöther, no início do século XX, enquanto alegoria da androcentria típica da academia daquele período, não pode ser considerada como uma questão solucionada. No decorrer das décadas, a misoginia deu lugar outras práticas, mas o seu impacto na desistência de muitas mulheres em entrar e progredir na carreira científica continua o mesmo Considerando Emmy como alegoria histórica, a perpetuação das práticas misóginas pode ser analisada ao de detalhar sobre o quadro participativo atual de mulheres que se dedicam a ciência.

Tomamos, então, como estudo de caso, as pesquisadoras Bolsistas do $\mathrm{CNPq}$.

O conjunto de pesquisadores do $\mathrm{CNPq}$ representa, em satisfatória medida, a liderança científica ativa no país, cobrindo todas as áreas, e recebem a seguinte classificação: pesquisador 1A (o mais alto escalão), 1B, 1C, 1D e Pesquisador nível 2 (o menor nível). As bolsas são distribuídas a partir de análise pelos pares, que são organizadas em comitês de áreas. Membros desses comitês são escolhidos entre os pesquisadores, e os resultados das análises são conduzidos aos setores administrativos, que podem impor cortes devido a limitações orçamentárias decorrentes de contingenciamentos impostos pelo governo. Entretanto, esses cortes respeitam em geral os critérios classificatórios das análises. Esse procedimento vem sendo desenvolvido e aprimorado desde a fundação dessa agência de fomento no fim da década de 1940.

Adquirir o título de Pesquisador do $C N P q$, se traduz em importante capital simbólico na luta por espaços políticos e científicos, dentro e fora da academia. Por exemplo, as cotas de bolsas para iniciação científica, em algumas universidades brasileiras, ficam a priori garantidas aos professores/pesquisadores com Bolsa de pesquisa (PQ). O restante é disputado pelos professores/pesquisadores sem Bolsa PQ. Medidas desta natureza, no interior da vida acadêmica, tornam a disputa pela Bolsa PQ muito acirrada e qualificam-se para tanto pesquisadores e pesquisadoras ativas e articuladas. Neste cenário, coletamos os dados dos Bolsistas PQ do CNPq, no período de 2001 a 2015, ou seja, por quinze (15) anos, distribuídos por gênero, apresentados na Figura 2 Esses resultados atualizam estudos prévios nessa direção 32 .

O surpreendente nos números da Figura 2 é que não refletem a participação das mulheres na ciência, pois um estudo estudo conduzido pela Elsevier 35 mostra que hoje as mulheres no Brasil respondem por cerca de $50 \%$ das publicações de artigos e pela maior parte das dissertações e teses 17.

Na Tabela 1] com dados extraídos da página da CAPES, estão apresentados os percentuais de participação geral de mulheres e homens, enquanto discentes, da pósgraduação brasileira. Na Tabela 2, com dados extraídos da página da CAPES, estão apresentados os percentuais de participação geral de mulheres e homens na docência de pós-graduação brasileira.

Esses números corroboram os dados da Elsevier, indicando paridade entre os discentes e um aumento de participação feminina na docência. No entanto, um estudo recente mostra que o percentual desta participação diminui à medida que se avança na carreira [36] e que na liderança em todas as áreas da ciência o percentual

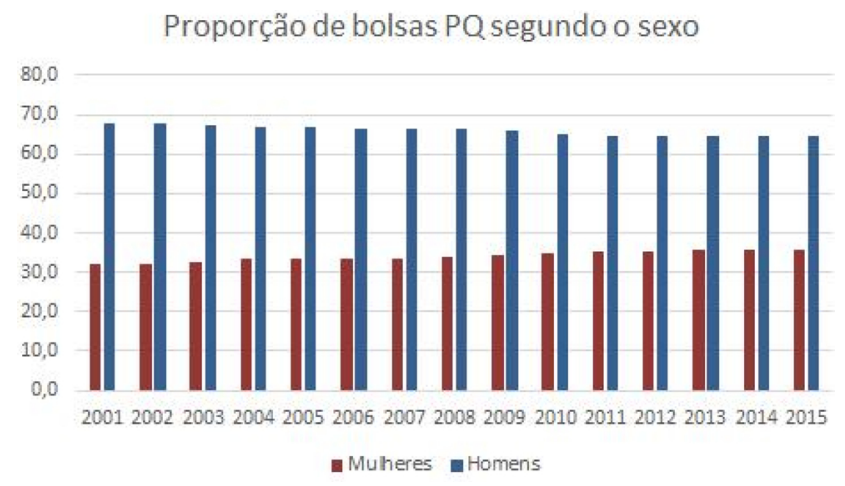

Figura 2: Pesquisadoras Bolsistas $P Q$. Fonte: www.cnpq.br; http://memoria.cnpq.br/series-historicas

Tabela 1: Porcentagem de discentes em todas as áreas de PósGraduação no Brasil. Fonte: Capes [17.

\begin{tabular}{ccc}
\hline Período & Homens & Mulheres \\
\hline 2005 & 50 & 50 \\
2010 & 49 & 51 \\
2015 & 47 & 53 \\
\hline
\end{tabular}

Tabela 2: Porcentagem de docentes em todas as áreas de PósGraduação no Brasil. Fonte: Capes [17.

\begin{tabular}{ccc}
\hline Período & Homens & Mulheres \\
\hline 2005 & 64 & 36 \\
2010 & 63 & 37 \\
2014 & 58 & 42 \\
\hline
\end{tabular}


de mulheres é muito baixo. Isso é acentuado em física e astronomia, área que se relaciona praticamente com todas as outras áreas do conhecimento e que funda as engenharias clássicas e contemporâneas, como as engenharias de materiais nano-estruturados 37]. A Tabela 3 mostra a participação discente na Pós-graduação brasileira na área de física e astronomia, que, de acordo com os dados coletados pela CAPES, ficaram em torno de $21 \%$ na última década. Estes números confirmam a baixa participação feminina mesmo como discentes nesta área e ilustra a tendência dessa ocupação se manter no mesmo patamar.

Na Tabela 4 os dados da participação das docentes na pós-graduação brasileira na área de física e astronomia estão apresentados, e fica em torno de $13,5 \%$ na última década de dados coletados pela CAPES. Estes números reforçam que as mulheres estão muito pouco representadas nos cursos de física e somem à medida que avançam na carreira.

Essa tendência ganha reforço com a análise dos dados de coordenadores dos Programas de Pós-Graduação e de coordenadores de área da CAPES [38, 39]. Os coordenadores de Programas de de Pós-Graduação, em todas as áreas, estão apresentados na Tabela 5 . Dados similares para a física e astronomia estão apresentados na Tabela 6. A Tabela 7 traz a porcentagem de coordenadoras de áreas da CAPES. Note que, embora haja alguma diferença quando comparados com os percentuais

Tabela 3: Porcentagem de discentes da área de Física e Astronomia. Fonte: Capes 17].

\begin{tabular}{ccc}
\hline Período & Homens & Mulheres \\
\hline 2005 & 80 & 20 \\
2010 & 79 & 21 \\
2015 & 78 & 22
\end{tabular}

Tabela 4: Porcentagem de docentes da area de Física e Astronomia. Fonte: Capes 17.

\begin{tabular}{ccc}
\hline Período & Homens & Mulheres \\
\hline 2005 & 86 & 14 \\
2010 & 87 & 13 \\
2015 & 87 & 13 \\
\hline
\end{tabular}

Tabela 5: Porcentagem de Coordenadores dos Programas de Pós-Graduação: todas as áreas. Fonte: Capes [17].

\begin{tabular}{ccc}
\hline Período & Homens & Mulheres \\
\hline $2006-2010$ & 60 & 40 \\
$2011-2015$ & 60 & 40 \\
\hline
\end{tabular}

Tabela 6: Porcentagem de coordenadores dos Programas de PósGraduação da área de Física e Astronomia. Fonte: Capes [17].

\begin{tabular}{ccc}
\hline Período & Homens & Mulheres \\
\hline $2006-2010$ & 87 & 13 \\
$2011-2015$ & 87,5 & 12,5 \\
\hline
\end{tabular}

Tabela 7: Porcentagem de coordenadores de área da CAPES todas as áreas. Fonte: Capes [38]

\begin{tabular}{ccc}
\hline Período & Homens & Mulheres \\
\hline $2011-2013$ & 59 & 41 \\
$2014-2016$ & 77 & 23 \\
\hline
\end{tabular}

ilustrados nas Tabela 2 e Tabela 5 , esses resultados poderiam sugerir que as mulheres estão galgando cargos na hierarquia da ciência, principalmente no que se refere à coordenação. Aqui, acurácia na análise é necessária, pois nos deparamos com um típico elemento de poder simbólico. Vejamos! Primeiro, é importante observar que a posição de coordenação requer trabalho exaustivo. Contudo, isso não vem necessariamente acompanhado de prestígio (de capital simbólico, portanto). Quando essa variável é considerada, a natureza da situação se torna mais transparente. $\mathrm{O}$ caso específico da física, uma das mais prestigiosas áreas da ciência, é revelador. A catalogação da prestigiosa coordenação de área da CAPES em física e astronomia, desde 2000 até o presente, é ocupada por homens: ver 17] (por dificuldade de acesso a dados na página da CAPES, não fomos capazes, até o momento, de retroagir esses dados ao período anterior do ano 2000). Ressalte-se que este resultado é consistente com aqueles descrevendo a participação feminina nas coordenações dos Programas de Pós-Graduação em física e astronomia, como apontamos. Pela Tabela 6 essa participação fica em torno de apenas $13 \%$.

A baixa presença de mulheres nesta disputa de posição de liderança se deve a dois fatores complementares. Mulheres tendem a subestimar a sua capacidade e sequer concorrem se não consideram que tem $100 \%$ de chances de ser efetivas no pleito 11,40, enquanto homens competem mesmo quando não têm todas as qualificações 41. Neste sentido, o número de mulheres solicitando bolsa é menor do aquele que potencialmente poderia estar apresentando projeto. Este fator, que começa a transparecer nas mulheres desde a juventude, a partir do entalhe cultural a que são submetidas, tem um agravante: os obstáculos adicionais que mulheres precisam transpor, como comentários desmotivadores e misóginos por parte de colegas 42]. O segundo fator é de natureza cultural, ou seja o viés inconsciente (naturalizado socialmente) [43], que não identifica as qualidades de liderança em mulheres.

A participação de mulheres em ciências no Brasil fica em torno de 33\%. Essa participação, descrita na Fígura 1, é uma reta estatisticamente de inclinação nula. Este tipo de resultado mostra a tendência de perpetuação minoritária das mulheres em ciência. Outros trabalhos corroboram este resultado, deixando transparente que a questão de gênero em ciência não é uma questão de "salas de banho". De fato, a história das mulheres em ciência, e em particular na física, é repleta de exemplos 14. É importante salientar que essa misoginia não reflete competência científica. Os resultados da Nöther, pela importância e extensão, são uma alegoria desse contexto 
meramente androcêntrico, como apontado anteriormente. Para precisar esses aspectos de que existe uma misoginia não associada à competência da pesquisadora, abordamos, na próxima seção, aqueles resultados do trabalho de pesquisa de Emmy em um caminho a permitir a discussão sobre a abrangência e a importância do teorema da Nöther para a física contemporânea.

\section{Resultados da Nöther revisitados}

Nesta seção revisitamos o teorema da Nöther, procurando uma apresentação pedagógica, que reflita o seu significado para as diversas áreas da física. O teorema assegura que a cada simetria está associado um invariante mecânico e é desenvolvido, usualmente, a partir da postulação da estrutura Lagrangiana-variacional. Para isso ampla e qualificada literatura está disponível [44]. Entretanto, para os propósitos de nossa discussão, enfatizamos a estrutura algébrica da mecânica, utilizando alguns resultados prévios 37,45 47.

Um programa mecânico (geral) pode ser definido a partir de um conjunto de elementos constitutivos, no qual o primeiro elemento é a existência do espaço e do tempo definidos a partir de um processo de mensuração [46, 47. Essas quantidades definem uma variedade de pontos $x=$ $\{\mathbf{x}, t\}$, tal que $\mathbf{x}$ designa vetores do $\mathbb{R}^{3}$, e $t \in \mathbb{R}^{1}$ descreve o tempo. A medida ocorre a partir de um laboratório, considerado como um locus no espaço-tempo $S$ e chamado de sistema de referência, que é definido pela relação com os outros sistemas de referência. A relação entre dois sistemas, $S$ e $\bar{S}$, se estabelece por um mapeamento $\Omega: S \rightarrow \bar{S}$, sendo $\Omega$ transformações especificadas pelo experimento. Sob condições amplas, vamos mostrar que $\Omega$ é grupo de simetria em $\mathcal{V}$, de tal modo que os fenômenos físicos em $S$ e $\bar{S}$ são descritos matematicamente da mesma maneira. O aspecto significativo é observar que a natureza de $\Omega$ fica imposta pelo experimento.

Um segundo elemento constitutivo de um projeto mecânico é a existência de um conjunto de variáveis mecânicas denotado por $\mathcal{V}=\{A, B, C, .$.$\} , entre as quais estão aque-$ las que descrevem a localização, velocidade, momentum, energia, dentre outras. Um conjunto básico dessas variáveis, a partir das quais todas as outras são construídas, é denominado de variáveis canônicas. As variáveis do conjunto gerado pelas variáveis canônicas são denominados observáveis do sitema físico e minimamente precisam possuir a estrutura de espaço linear, para garantir, por exemplo, a soma de velocidades ou de forças, e assim por diante. Para permitir o produto dos observáveis mecânicos, o espaço linear $\mathcal{V}$ é equipado com uma estrutura de álgebra associativa. Isto garante, por exemplo, a expansão em série de potências de qualquer quantidade em termos das variáveis canônicas. Esta álgebra associativa é designada pelo produto $\circ$, em $\mathcal{V}$, ou seja, $\circ: \mathcal{V} \rightarrow \mathcal{V}$, de modo que

$$
A \circ B=A B=C \in \mathcal{V},
$$

para todo $A, B \in \mathcal{V} 45,46$.

Outro elemento constitutivo de um projeto mecânico é o conceito de estado mecânico $(\mathcal{E})$, que pode ser definido como um conjunto de informações de tal forma a considerar os seguintes aspectos. (i) Estabelecer a localização do sistema físico no espaço e no tempo. (ii) Prover elementos do movimento; isto é, fornecer uma ou mais característica intrínsica do movimento do sistema, escolhida como um conceito primitivo. (iii) Dado um observável $\mathcal{O}$, deve existir uma regra a ser estabelecida experimentalmente de modo que $\mathcal{E}(\mathcal{O}) \in \mathbb{R}^{n}$, correspondendo ao processo de mensuração 47.

Vamos considerar o que se chama de descrição de Heisenberg, de tal modo que o estado do sistema fica fixado por mudanças parametrizadas dos sistemas de referências. Assim somente os observáveis estão dados a mudar. Essas mudanças de sistemas de coordenadas estão conectadas à identidade, ou seja passíveis de serem descritas infinitesimalmente, devido à mudança paramétrica. Os parâmetros podem ser tempo, ângulos, etc. Desse modo, a modificação em um observável $A \in \mathcal{V}$ é dada pela variação paramétrica de um quantidade $\tau \in \mathbb{R}$, de modo que

$$
\delta A=A(\tau+\delta \tau)-A(\tau)
$$

Esta mudança pode ser assegurada pela existência de outra variável mecânica, um gerador, $B \in \mathcal{V}$. Isto fica acomodado ao se introduzir uma aplicação $():, \mathcal{V} \times \mathcal{V} \rightarrow \mathcal{V}$, com $\delta A=(A, B) \delta \tau$. Observe que a operação $\delta A$ é linear, ou seja $\delta(A+b B)=\delta A+b \delta B$, com $b \in \mathbb{R}$. Então devemos considerar que $($, ) seja também linear, por consistência. Nesse sentido, o mapeamento ( , ) torna o espaço vetorial $\mathcal{V}$ uma álgebra linear, cuja estrutura devemos encontrar a partir de elementos experimentais 45,46.

Cada elemento de $\mathcal{V}$ pode ser interpretado, simultaneamente, como um observável e um gerador de mudanças em outro elementos de $\mathcal{V}$. Contudo, devemos impor a restrição física de que um gerador, digamos $C$, não pode impor variações mecânicas sobre si mesmo. Ou seja, devese requerer que $(C, C)=0$. Considerando $C=A+B$, e que $($, ) é uma operação linear, então temos

$$
(A+B, A+B)=(A, A)+(A, B)+(B, A)+(B, B) .
$$

Como por construção $(A, A)=(B, B)=0$, segue que

$$
(A, B)=-(B, A)
$$

ou seja, a aplicação linear ( , ) é antissimétrica.

Uma vez que $\mathcal{V}$ é também uma álgebra associativa, podemos escrever $C=A B$ e calcular uma mudança infinitesimal de $C$, que fica dada por $\delta C=(\delta A) B+$ $A(\delta B)$. Considerando que esta mudança possa ser gerada por uma variável $D$ e especificada por um parâmetro $\tau$, segue então

$$
\begin{aligned}
\delta A & =(A, D) \delta \tau \\
\delta B & =(B, D) \delta \tau \\
\delta C & =(C, D) \delta \tau .
\end{aligned}
$$


Isto conduz a

$$
(A B, C)=(A, C) B+A(B, C) .
$$

Ou seja, a associação do produto ( , ) com o produto associativo, o, é uma derivação, ou a regra de Leibniz.

Fisicamente devemos ainda impor as seguintes condições sobre a aplicação ( , ). Considere $D \in \mathcal{V}$ de modo que

$$
D(\tau)=(A(\tau), B(\tau)) .
$$

Logo, por uma questão de estabilidade das condições físicas, uma mudança infinitesimal no parâmetro $\tau$, ou seja $\delta \tau$, conduz a

$$
D(\tau+\delta \tau)=(A(\tau+\delta \tau), B(\tau+\delta \tau)) .
$$

Escrevendo $\delta A=(A, C) \delta \tau, \delta B=(B, C) \delta \tau$ e $\delta D=$ $(D, C) \delta \tau$, após algum cálculo, segue

$$
(A,(B, C))+(C,(A, B))+(B,(C, A))=0,
$$

que é conhecida como identidade Jacobi. Logo, a aplicação ( , ) é um produto de Lie, e $\mathcal{V}$ é uma álgebra Lie-algebra com derivação 45, 46], e portanto ligada a um grupo de simetria descrevendo as mudanças dos observáveis físicos.

Esses resultados são gerais o suficiente para se extrair várias informações sobre os sistemas mecânicos. Assim, por exemplo, se uma grandeza $I(\mathcal{E})$ for invariante pela transformação $\Omega$, ou seja transformações de simetria a serem especificadas, então $I(\mathcal{E})$ é um invariante sobre um grupo de Lie. Em outras palavras, $I(\mathcal{E})$ pode ser escrita em associação com uma corrente conservada, o que expressa o conteúdo do teorema de Nöther. Vamos, a seguir, considerar um exemplo, especificando a natureza do grupo de simetria $\Omega$. Trataremos apenas em um tipo simetrias do espaço-tempo, a que descreve sistemas relativísticos. Entretanto, outras simetrias do espaço-tempo, como as de Galilei ou de De Sitter, e também as simetrias de calibre podem ser consideradas 46, 47.

Considere $I(\mathcal{E})$ um invariante relativístico. Neste caso $\Omega$ é o grupo de Lorentz 46, 47], e assim segue que

$$
\Omega I(\mathcal{E}) \Omega^{-1}=I(\mathcal{E}) .
$$

Esta invariância é resultado de uma exigência experimental, imposta sobre o sistema em estudo. Infinitesimalmente podemos escrever

$$
d I(\mathcal{E})=\rho_{I} d x^{1} d x^{2} d x^{3},
$$

que define a noção de densidade, $\rho_{I}$, do invariante $I(\mathcal{E})$. Estamos adotando a métrica, $g$, diagonal, tal que diagg $=$ $(1,-1,-1,-1)$, e $d x=\left(d x^{\mu}\right)=\left(d x^{0}, d x^{1} d x^{2} d x^{3}\right)$, com $d x^{0}=c d t$, sendo $\mu=0,1,2,3 ; t$ é o tempo e $c$ é a velocidade da luz.

Como $d I(\mathcal{E})$ é um invariante de Lorentz, segue da Eq. (3) que $\rho_{I}$ se transforma como a componente tipotempo de um quadrivetor. Além disso, sendo $\mathbf{v}=\left(v^{1}, v^{2}, v^{3}\right)$ a velocidade de $d I(\mathcal{E})$, podemos definir o vetor Euclidiano densidade de corrente

$$
j^{k}=\rho_{I} v^{k} ; k=1,2,3 .
$$

De modo outro,

$$
j^{k}=j^{0} \frac{\partial x^{k}}{\partial x^{0}}
$$

com $j^{0}=c \rho_{I}$ sendo a componente tipo-tempo da 4corrente, que é escrita como

$$
\left(j^{\mu}\right)=\left(j^{0}, j^{1}, j^{2}, j^{3}\right)
$$

Como $d I(\mathcal{E})$ é Lorentz-invariante, localmente isso se traduz na equação da continuidade, isto é

$$
\partial_{\mu} j^{\mu}=0
$$

que significa uma corrente conservada, como prescreve o teorema de Nöther.

É importante observar que essa estrutura de álgebra de Lie é deduzida independentemente da natureza de $\Omega$ que pode ser não-relativística (neste caso, trata-se do grupo de Galilei) ou relativística (ou seja, o grupo de "Lorentz"). Além disso, o sistema físico pode ser um campo ou um conjunto de pontos materiais 47. Se o sistema for um campo, as simetrias podem incluir transformações internas. Por exemplo, tomando a simetria de rotação aplicada à componentes do campo eletromagnético conduz ao efeito de polarização da luz. A mesma simetria aplicada a componentes do campo de Schrödinger ou de Dirac conduz ao conceito de spin. Generalizações levam ao conceito de cores, sabores e às interações fundamentais, descritas a partir dos campos de calibre (gauge). Com os invariantes de Nöther, as partículas básicas são então classificadas. Por exemplo, o elétron fica caracterizado por suas características invariantes: massa, carga e spin. É essa abrangência que subjaz ao teorema de Nöther, e disto resulta sua relevância enquanto um construto para toda a física contemporânea.

\section{Conclusões}

Neste trabalho analisamos os elementos da biografia de Emmy Nöther com ênfase na discriminação que sofrera em sua vida acadêmica, no início do Século XX, a despeito da importância reconhecida de seus trabalhos, como a dedução dos teoremas de Nöther. Sua situação, proveniente de uma prática que tem raízes profundas em uma sociedade patriarcal, foi entã considerada como referência histórica para se conduzir uma análise da condição da mulher pesquisadora no Brasil nesse início do Século XXI.

Esta análise se baseia na hipótese de que a misoginia sofrida por Emmy no começo do século XX tem características semelhantes à que afasta hoje mulheres da ciência. Para isso, abordamos, inicialmente, a participação de pesquisadoras no montante de bolsas de produtividade em 
pesquisa do CNPq, tendo em vista que essas bolsas definem uma parcela significativa das lideranças científicas ativas no país. Esses dados foram implementados com outros obtidos a partir do portal da CAPES. O principal resultado é a constatação de que embora a situação tenha apenas melhorado, com relação ao período histórico da Professora Nöther, a conotação androcêntrica está longe de ser resolvida: a curva de participação das mulheres bolsistas do CNPq, numa escala de tempo de 15 anos (2001 a 2015) é descrita por retas com inclinação estatisticamente nula. Para a discussação da abrangência e da importância dos teoremas, conduzimos a apresentação dos resultados através de um procedimento algébrico, o que viabilizou nossos propósitos, sem perder de vista elementos pedagógicos. Essa análise da abrangêcia e importância, bem como da situação de discriminação a que foi submetida Emmy Nöther no início do Século XX, mostra que a androcentria no campo científico é marca registrada, com tendência a perpetuação.

Evocando a Professora Emmy Nöther como no seu teorema, atestando que para um sistema invariante por uma simetria há uma lei de conservação associada, talvez pudéssemos afirmar que a invariância da misoginia por uma simetria descrevendo translações temporais leva a se conservarem ao longo do tempo o machismo e a exclusão social. Somente uma quebra dessa simetria faria a exclusão deixar de existir. Isto significa que, sem mudanças nas políticas gestoras, a conclamada equidade de gêneros em ciências pode não ocorrer. Essa quebra de simetria deve ser conduzida através de alterações estruturais. Assim, possivelmente, demande um novo modo de produção de bens simbólicos, que possam apontar para uma nova divisão do trabalho científico. Esses aspectos, assim como uma análise mais detalhada do mecanismo de perpetuação da misoginia no campo científico, serão abordados em outro trabalho.

\section{Agradecimentos}

Este trabalho recebeu apoio financeiro parcial do $\mathrm{CNPq}$ e da CAPES.

\section{Referências}

[1] E. Nöther, Journal für die Reine und Angewandte Mathematik 134, 23 (1908).

[2] A. Ribeiro-Filho, in: Mulheres na física: Casos históricos, panorama e perspectivas, organizado por E.B. Saitovitch, M.C.B. Barbosa, S. de Pinho, R.Z. Funchal e A.E. Santana (LF Editorial, São Paulo, 2015), p. 31.

[3] M.K. Smith, Emmy Noether: A Tribute to Her Life and Work (Marcel Dekker, New York, 1981).

[4] A. Dick, A. Emmy Noether: 1882-1935 (Birkhauser, Boston, 1981).

[5] M.B.W. Tent, Emmy Noether: The Mother of Modern Algebra (AK Peters, Wellesley, 2008).

[6] M. Patrão, e-Boletim da Física 4, 2102-1 (2015).

[7] cwp.library.ucla.edu/articles/noether.asg/noether.html.
[8] https://blog.nli.org.il/en/noether/.

[9] E.B. Saitovitch, M.C.B. Barbosa, S. de Pinho, R.Z. Funchal e A.E. Santana, Mulheres na Física: casos históricos, panorama e perspectivas (LF Editorial, São Paulo, 2015).

[10] M.C. Barbosa e B.S. Lima, in: Trabalhadoras: Análise da Feminização das Profissões e Ocupações, organizado por Silvia Cristina Yannoulas (Editora Abaré, Brasília, 2013).

[11] https://web.stanford.edu/niederle/nv.annualreview.print.pdf.

[12] R. Ivie e K.N. Ray, Women in Physics and Atronomy, disponível em https://www.aip.org/sites/default/ files/statistics/women/women-pa-05.pdf

[13] D.A. Agrello e R. Garg, Rev. Bras. Ens. Fis. 31, 1305 (2009).

[14] N. Byers e G. Williams, Out of the Shadows: Contributions of Twentieth-Century Women to Physics (Cambridge University Press, New York, 2006).

[15] Gender gap in physics among highest in science, disponível em https://physicsworld.com/a/gender-gap-inphysics-amongst-highest-in-science/.

[16] E.B. Saitovitch, M.C. Barbosa, R.Z. Funchal, S.T.R. de Pinho e A.E. Santana, A Exclusão Invisivel, disponível em http://www.sbf1.sbfisica.org.br/boletim1/ msg260.htm

[17] Plataforma Sucupira, disponível em https://sucupira.capes.gov.br/sucupira/, acessado em 10/12/2018.

[18] P. Bourdieu, A Dominação Masculina (Bertrand Brasil, Rio de Janeiro, 1999).

[19] L. Abramo, Trabajo Decente y Equidad de Género en América Latina (Oficina Internacional del Trabajo, Santiago, 2006).

[20] I.M. Young, Justice and Politics of Difference (Princeton University Press, Princeton, 1990).

[21] N. Elias, A Sociedade dos Indivíduos (Fayard, Paris, 1990).

[22] D. Diniz, S. Buglione e R.R. Rios, Entre a Dúvida e o Dogma (Letras Livres, Brasília, 2006).

[23] W. Rogers e A. Ballantyne, in: Ética em Pesquisa: Temas Globais, editado por D. Diniz, A. Sugai, D. Guilhem e F. Squinca (EdUnB, Brasília, 2008).

[24] C. Schwantes, in: Mulher e Literatura - 25 anos: Raízes e Rumos, editado por C. Stevens (Editora Mulheres, Florianópolis, 2010).

[25] K. Braga e E. Nascimento, Bibliografia Maria da Penha: Violência Contra a Mulher no Brasil, editado por D. Diniz (Letras Livres e EdUnB, Brasília, 2006).

[26] E.B. Saitovitch, M.C.B. Barbosa, S.T.R. de Pinho e A.E. Santana, e-Boletim da Física 3, 003-1 (2014).

[27] A.E. Santana, in: Estudos Feministas e de Gênero: Articulações e Perspectivas, organizado por C. Stevens, S. Rodrigues de Oliveira e V. Zanello (Editora Mulheres, Florianópolis, 2014), p. 327.

[28] T.M.F. Mourão, Mulheres no Topo da Carreira (Secretaria Especial de Políticas para as Mulheres, Brasília, 2006).

[29] F.M. Paz, Senadoras: Dados Biográficos (Secretaria de Arquivo, Senado Federal, Brasília, 2004).

[30] L.L. Barsted, in: Autonomia Econômica e Empoderamento da Mulher (Fundação Alexandre de Gusmão, Ministério das Relações Exteriores, Brasília, 2011). 
[31] A.P. Ibaldo, in: Estudos Feministas e de Gênero: Articulações e Perspectivas, organizado por C. Stevens, S. Rodrigues de Oliveira e V. Zanello (Editora Mulheres, Florianópolis, 2014), p. 313.

[32] E.B. Saitovitch, B.S. Lima e M.C. Barbosa, in: Mulheres na física: Casos históricos, panorama e perspectivas, organizado por E.B. Saitovitch, M.C.B. Barbosa, S. de Pinho, R.Z. Funchal e A.E. Santana (LF Editorial, São Paulo, 2015), p. 245.

[33] C. Ashcraft, B. McLain e E. Eger, Women in Tech: The Facts, disponível em https: //www.ncwit.org/sites/default/files/resources/ womenintech_facts_fullreport_05132016.pdf

[34] https://cds.cern.ch/record/272289/files/ 9411110.pdf.

[35] https://www.elsevier.com/research-intelligence/ campaigns/gender-17

[36] N.C. Ferrari, R. Martell, D.H. Okido, G. Romanzini, V. Magnan, M.C. Barbosa e C. Brito, Anais de Academia Brasileira de Ciências 90, 2243 (2018).

[37] L.A.C. Malbouisson e A.E. Santana, Ciência e Sociedade 2, 1 (2014).

[38] BRASIL. Portaria n.o 47, de 4 de abril de 2011. Brasíla, 2011. Disponível em: https://www.capes.gov.br/ images/stories/download/avaliacao/avaliacao-n/ Portaria-capes-47-2011.pdf.

[39] BRASIL. Portaria n.o 107, de 14 de agosto de 2014. Brasíla, 2014. Disponível em: https://www.capes.gov br/images/stories/download/legislacao/1882014portaria-n-107-de-14-8-2014.pdf

[40] E.M. Marshman, Z.Y. Kalender, T. Nokes-Malach, C. Schunn e C. Singh, Phys. Rev. Phys. Educ. Res. 14, 020123 (2018).

[41] https://hbr.org/2014/08/why-women-dont-apply-forjobs-unless-theyre-100-qualified.

[42] https://www.bcg.com/pt-br/publications/ 2017/people-organization-leadership-changedispelling-the-myths-of-the-gender-ambitiongap.aspx.

[43] Www.pnas.org/cgi/doi/10.1073/pnas.1510649112

[44] L.H. Reyder, Quantum Field Theory (Cambridge University Cambridge, 1996).

[45] H.Q. Plácido, R. Bunchaft e A.E. Santana, Hadronic J. 15, 225 (1992).

[46] A.E. Santana e S. Simon, J. Mod. Phys. 6, 58 (2015).

[47] A.E. Santana, Rev. Bras. Ens. Fis. 41, e20180145 (2019). 